\title{
SYMMETRIZATION OF RINGS IN SPACE
}

\author{
BY \\ F. W. GEHRING
}

\section{INTRODUCTION}

The purpose of this paper is to obtain a pair of upper bounds for the moduli of rings in space by means of symmetrization. That is with each ring $R$ we associate a second ring $R^{\prime}$, obtained by symmetrizing $R$, for which the fundamental inequality

$$
\bmod R \leqq \bmod R^{\prime}
$$

holds. We then estimate mod $R^{\prime}$ either by means of the space analogues of the Grötzsch and Teichmüller rings or by means of spherical annuli.

The two bounds we obtain are given in Theorem 3 of $\$ 17$ and in Theorem 4 of \$22. In a later paper we will show how these upper bounds can be used to derive a number of important distortion theorems for quasiconformal mappings in space. For a summary of these results see [4].

\section{PRELIMINARY RESULTS}

1. Notation. We consider here sets in finite Euclidean 3-space. Points will be designated by capital letters $P$ and $Q$ or by small letters $x$ and $y$. In the latter case $x_{1}, x_{2}$ and $x_{3}$ will represent the coordinates for $x$ and similarly for $y$. Points are treated as vectors and $|P|$ and $|x|$ will denote the norms of $P$ and $x$, respectively.

Given a set $E$ we let $\partial E$ denote its boundary, $\mathbf{C} E$ its complement, $\bar{E}$ its closure and int $E$ its interior. The Lebesgue 3-dimensional measure of $E$ will be written as $m(E)$ and, unless otherwise stated, a.e. will be taken with respect to $m$. By the area of $E$ we will mean the Hausdorff 2-dimensional measure of $E$ defined as follows:

$$
\Lambda^{2}(E)=\lim _{a \rightarrow 0+}\left(\inf \sum_{U \in \mathcal{U}} \frac{\pi}{4} d(U)^{2}\right) .
$$

Here, for each $a>0$, the infimum is taken over all countable coverings $u$ of $E$ by sets $U$ with diameters $d(U)<a$. When $E$ is a plane set, this reduces to the Lebesgue 2-dimensional measure. Finally by the length of $E$ we mean the Hausdorff 1-dimensional or linear measure $\Lambda(E)$.

2. Modulus of a ring. A ring is defined as a domain whose complement consists of two components, one of which is unbounded. Given a ring $R$ we et $C_{0}$ and $C_{1}$ denote, respectively, the bounded and unbounded components

Received by the editors February 16, 1961 . 
of $C_{R}$. We further let $B_{0}=\partial C_{0}$ and $B_{1}=\partial C_{1}$. These are the components of $\partial R$.

Now let $u$ be any function which is continuously differentiable in $R$ and has boundary values 0 on $B_{0}$ and 1 on $B_{1}$. (When $R$ is unbounded, this will mean that $u(x) \rightarrow 1$ as $|x| \rightarrow \infty$ in $R$.) Then following Loewner [7] we define the conformal capacity of $R$ as

$$
\Gamma(R)=\inf _{u}|\nabla u|^{3} d \omega,
$$

where the infimum is taken over all such functions $u .\left(^{(1)}\right.$ The modulus of $R$ is then defined by

$$
\bmod R=\left(\frac{4 \pi}{\Gamma(R)}\right)^{1 / 2} .
$$

This is the space analogue of the modulus of a plane ring usually defined with the aid of conformal mapping.

As an example we calculate the modulus of the spherical annulus $a<|x|$ $<b$. For this let $u$ be continuously differentiable in $R$ with boundary values 0 on $|x|=a$ and 1 on $|x|=b$. Then integrating along a radius and applying Hölder's inequality yields

$$
1 \leqq\left(\int_{a}^{b}|\nabla u| d r\right)^{3} \leqq\left(\int_{a}^{b}|\nabla u|^{3} r^{2} d r\right)\left(\log \frac{b}{a}\right)^{2} .
$$

Hence

$$
\frac{4 \pi}{(\log (b / a))^{2}} \leqq \int_{R}|\nabla u|^{3} d \omega .
$$

On the other hand choosing

$$
u(x)=\frac{\log (|x| / a)}{\log (b / a)}
$$

yields

$$
\frac{4 \pi}{(\log (b / a))^{2}}=\int_{R}|\nabla u|^{3} d \omega
$$

We conclude that

$$
\Gamma(R)=\frac{4 \pi}{(\log (b / a))^{2}}
$$

whence

(l) $\nabla u$ denotes the vector $\left(\partial u / \partial x_{1}, \partial u / \partial x_{2}, \partial u / \partial x_{3}\right)$. 


$$
\bmod R=\log \frac{b}{a}
$$

3. Admissible functions. It is convenient in defining the conformal capacity, to relax the differentiability requirements for $u$ and take the infimum in (2) over a slightly larger class of functions. We say that a function $u$ is ACL or absolutely continuous on lines in a domain $D$ if, given any sphere $U$ with $\bar{U} \subset D, u$ is absolutely continuous on almost all line segments in $U$ which are parallel to the coordinate axes. If $u$ is continuous and ACL in a ring $R$, then $u$ has partial derivatives a.e. in $R$. If, in addition, $u$ has boundary values 0 on $B_{0}$ and 1 on $B_{1}$, we say that $u$ is an admissible function for the ring $R$.

Leмma 1. If $u$ is admissible for a ring $R$, then

$$
\Gamma(R) \leqq \int_{R}|\nabla u|^{3} d \omega .
$$

Proof. We may assume that $|\nabla u|$ is $L^{3}$-integrable over $R$, for otherwise there is nothing to prove. Next fix $0<a<1 / 2$, let

$$
v= \begin{cases}0 & \text { if } u<a, \\ \frac{u-a}{1-2 a} & \text { if } a \leqq u \leqq 1-a, \\ 1 & \text { if } 1-a<u,\end{cases}
$$

and extend $v$ to be 0 on $C_{0}$ and 1 on $C_{1}$. The set where $a \leqq u \leqq 1-a$ is a compact subset of $R$ and lies at a distance $b$ from $\partial R$. Let $U$ be the sphere $|y|<c$, $c<b$, and let

$$
w(x)=\frac{1}{m(U)} \int_{U} v(x+y) d \omega .
$$

This function is continuously differentiable in $R$ and has boundary values 0 on $B_{0}$ and 1 on $B_{1}$. From (6) we see that $v$ is ACL everywhere and, with Hölder's inequality, that $|\nabla v|$ is $L$-integrable over each compact set. Hence we can apply Fubini's theorem to conclude that

$$
\nabla w(x)=\frac{1}{m(U)} \int_{U} \nabla v(x+y) d \omega
$$

for each $x \in R$. Then applying Minkowski's inequality twice we obtain

$$
\left(\int_{R}|\nabla w(x)|^{3} d \omega\right)^{1 / 3} \leqq \frac{1}{m(U)} \int_{U}\left(\int_{R}|\nabla v(x+y)|^{3} d \omega\right)^{1 / 3} d \omega .
$$

The inner integral on the right is majorized by 


$$
(1-2 a)^{-3} \int_{R}|\nabla u(x)|^{8} d \omega
$$

for each $y \in U$. Hence

$$
\int_{R}|\nabla w|^{3} d \omega \leqq(1-2 a)^{-3} \int_{R}|\nabla u|^{3} d \omega,
$$

and (2) yields

$$
\Gamma(R) \leqq(1-2 a)^{-3} \int_{R}|\nabla u|^{3} d \omega
$$

The desired inequality (5) is now obtained by letting $a \rightarrow 0$.

4. Extremal function. From Lemma 1 it follows we can enlarge the class of competing functions in the definition of $\Gamma(R)$ to include those which are admissible for $R$, that is

$$
\Gamma(R)=\inf _{u} \int_{R}|\nabla u|^{3} d \omega
$$

where now the infimum is taken over all admissible functions $u$. If $R$ has nondegenerate boundary components, we can show that there exists an extremal admissible function $u$ for which

$$
\Gamma(R)=\int_{R}|\nabla u|^{3} d \omega .
$$

This function is unique and we call it the extremal function for $R$. It is the space analogue for the harmonic measure whose Dirichlet integral yields the electrostatic capacity of a plane ring. Next if, for each compact set $E \subset R$, a positive constant $M$ exists such that

$$
1 / M \leqq|\nabla u| \leqq M
$$

a.e. in $E$, we can show that the extremal function $u$ is real analytic and

$$
\operatorname{div}(|\nabla u| \nabla u)=0
$$

everywhere in $R$. Proofs for these results will appear in a later paper.

5. Remark. The proof for Lemma 1 also implies that

$$
\Gamma(R)=\inf _{w}|\nabla w|^{3} d \omega
$$

where $w$ is everywhere continuously differentiable, $w$ is 0 on $C_{0}$ and 1 on $C_{1}$, $0 \leqq w \leqq 1$ in $R$ and $\nabla w$ vanishes off a compact subset of $R$; for the function defined in (7) has these properties. Hence if we choose $u$ so that 


$$
\int_{R}|\nabla u|^{3} d \omega<\Gamma(R)+\epsilon
$$

letting $a \rightarrow 0$ in (8) yields

$$
\Gamma(R) \leqq \inf _{w} \int_{R}|\nabla w|^{8} d \omega \leqq \Gamma(R)+\epsilon,
$$

from which (9) follows.

6. Monotoneity and superadditivity properties. A set $E$ is said to separate the boundary components of a ring $R$ if $E \subset R$ and each component of $\mathbf{C} E$ contains at most one component of $\mathbf{C} R$.

We can now use the above remark to establish the following monotoneity and superadditivity properties for the moduli of rings.

LEMмA 2. If $R^{\prime}$ is a ring which separates the boundary components of $R$, then $\bmod R \geqq \bmod R^{\prime}$.

If $R_{1}, R_{2}, \cdots, R_{n}$ are disjoint rings each of which separates the boundary components of $R$, then

$$
\bmod R \geqq \sum_{1}^{n} \bmod R_{i}
$$

Proof. We consider only the proof for (10). For each ring $R_{i}$ let $u_{i}$ be everywhere continuously differentiable, let $u_{i}$ be 0 and 1 , respectively, on $C_{0, i}$ and $C_{1, i}$, the components of $\mathbf{C} R_{i}$, and let $\nabla u_{i}$ vanish off a compact subset of $R_{i}$. Next set

$$
u=\sum_{1}^{n} a_{i} u_{i} \quad \text { where } \quad \sum_{1}^{n} a_{i}=1, \quad a_{i} \geqq 0
$$

Then

$$
\int_{R}|\nabla u|^{3} d \omega=\sum_{1}^{n} a_{i}^{3} \int_{R_{i}}\left|\nabla u_{i}\right|^{3} d \omega .
$$

Since $C_{0} \subset C_{0, i}$ and $C_{1} \subset C_{1, i}$ for all $i, u$ is admissible for $R$ and taking infimums over all such $u_{i}$ gives

$$
\Gamma(R) \leqq \sum_{1}^{n} a_{i}^{3} \Gamma\left(R_{i}\right)
$$

If $\Gamma\left(R_{i}\right)>0$ for all $i$, setting

$$
a_{i}=\Gamma\left(R_{i}\right)^{-1 / 2}\left(\sum_{1}^{n} \Gamma\left(R_{j}\right)^{-1 / 2}\right)^{-1}
$$


in (11) yields (10). If some $\Gamma\left(R_{i}\right)=0$, then setting $a_{i}=1$ and $a_{j}=0$ for $j \neq i$ again yields (10).

7. Simple admissible functions. In proving that the modulus of a ring $R$ is not decreased under symmetrization, we will want to consider admissible functions whose level surfaces are particularly well behaved.

Let $w$ be one of the functions considered in $\$ 5$. The set where $0<w<1$ is bounded and lies at a distance $b$ from $\mathbf{C} R$. Now consider a decomposition of the space into congruent tetrahedra $\{T\}$ with diameter $c<b$. Then define a new function $u$ so that $u$ is a linear function of the coordinate variables in each tetrahedron and so that $u=w$ on the vertices of each tetrahedron. Then $u$ is admissible for $R$ and

$$
\lim _{c \rightarrow 0} \int_{R}|\nabla u|^{3} d \omega=\int_{R}|\nabla w|^{3} d \omega .
$$

Clearly $0 \leqq u \leqq 1$. Now fix $a, 0 \leqq a<1$, and let $F$ be the set where $u \leqq a$ and $\Sigma$ the set where $u=a$. Then $F$ is a closed polyhedron, that is the union of a finite number of closed (possibly degenerate) tetrahedra, and $\partial F \subset \Sigma$. If, in addition, we choose $a$ to be different from the finite set of values assumed by $w$, and hence by $u$, on the vertices of the tetrahedra $\{T\}$, then it is easy to see that each point of $\Sigma$ is a boundary point of $F$, whence $\partial F=\Sigma$.

We say that any such function $u$ is a simple admissible function for $R$. Combining (12) and the result of $\$ 5$ then shows that

$$
\Gamma(R)=\inf _{u} \int_{R}|\nabla u|^{3} d \omega
$$

where the infimum is taken over all simple admissible functions $u$.

\section{SPHERICAL SYMMETRIZATION( $\left.{ }^{2}\right)$}

8. Spherical symmetrization of rings. Given an open set $G$ we define a second set $G^{*}$, the spherical symmetrization of $G$, as follows. For each $r \geqq 0$ let $S=S(r)$ denote the spherical surface $|x|=r$. Then $S \cap G^{*}$ is to be null if and only if $S \cap G$ is null. Next $S \subset G^{*}$ if and only if $S \subset G$. For the remaining case let $G$ meet $S$ in a set whose area is $A$. Then $0<A \leqq 4 \pi r^{2}$ and $G^{*}$ is to meet $S$ in a single open spherical cap of area $A$ with center at $(-r, 0,0)$; when $A=4 \pi r^{2}$, this cap will consist of $S$ minus the point $(r, 0,0)$. It is easy to see that $G^{*}$ is itself an open set and that $\mathbf{C} G^{*}$ is connected whenever $\mathbf{C} G$ is.

Next given a closed set $F$ we define $F^{*}$ exactly as above except in the last case. Here $0 \leqq A<4 \pi r^{2}$ and $F^{*}$ is to meet $S$ in a closed spherical cap of area $A$ with center at $(-r, 0,0)$; when $A=0$, this cap will consist only of the center point $(-r, 0,0)$. Then $F^{*}$ is closed and $F^{*}$ is connected whenever $F$ is.

(2) This method of symmetrization is discussed in [8, pp. 205-210]. 
Now let $R$ be a ring. Then $R \cup C_{0}$ is open, $C_{0}$ is closed and we define the spherical symmetrization of $R$ as

$$
R^{*}=\left(R \cup C_{0}\right)^{*}-C_{0}^{*}
$$

It is easy to verify that $R^{*}$ is again a ring and the purpose of this section is to show that $R^{*}$ enjoys the following extremal property.

THEOREM 1. $\bmod R \leqq \bmod R^{*}$.

The proof for Theorem 1 requires a preliminary study of some geometrical properties of spherically symmetrized sets.

9. Surface area under spherical symmetrization. It is easy to see that the measure of a closed set $F$ is preserved under spherical symmetrization. For if $A$ denotes the area of $S \cap F$, then

$$
m(F)=\int_{0}^{\infty} A(r) d r=m\left(F^{*}\right)
$$

as desired. The following result shows that in certain cases we can say something about what happens to the area of $\partial F$ under spherical symmetrization.

LеммA 3. If $F$ is a closed polyhedron and if $F^{*}$ is the spherical symmetrization of $F$, then $\partial F^{*}$ is a surface of revolution whose area does not exceed that of $\partial F$.

Proof. Let $\sigma=\sigma(r)$ and $\sigma^{*}=\sigma^{*}(r)$ denote the area of the parts of $\partial F$ and $\partial F^{*}$ contained in $|x| \leqq r$ for $r \geqq 0$. We shall show that

$$
\sigma^{*}\left(r_{2}\right)-\sigma^{*}\left(r_{1}\right) \leqq \sigma\left(r_{2}\right)-\sigma\left(r_{1}\right)
$$

for all $0 \leqq r_{1}<r_{2}<\infty$.

Now let $A$ be the area of $S \cap F$ where $S$ is the surface $|x|=r$. Then $A(r)$ is continuous and satisfies a uniform Lipschitz condition. This is clear when $F$ is a closed tetrahedron and hence the result follows when $F$ is a closed polyhedron. Since int $F$ and $\mathbf{C} F$ each have a finite number of components, the sets where $A>0$ and where $A<4 \pi r^{2}$ each consist of a finite number of open intervals. Hence the set where $0<A<4 \pi r^{2}$ is the finite union of open disjoint intervals $I$. Since $\sigma^{*}$ is constant in each complementary interval and since both $\sigma$ and $\sigma^{*}$ are continuous in $r$, it will be sufficient to establish (13) for the case where $r_{1}$ and $r_{2}$ belong to one of the intervals $I$.

Let $J$ denote the closed interval $r_{1} \leqq r \leqq r_{2}$ and for each point $x$ with $|x|>0$ let $\phi$ denote the angle between the radius to $x$ and the negative half of the $x_{1}$-axis. Then $\partial F^{*}$ has the representation

$$
\phi=f(r)=\arccos \left(1-\frac{A}{2 \pi r^{2}}\right), \quad|x|=r
$$

for $r \in J$. Since $A$ is bounded away from 0 and $4 \pi r^{2}$ in $J, f$ satisfies a Lipschitz condition there and it is not difficult to show directly that 


$$
\sigma^{*}\left(r_{2}\right)-\sigma^{*}\left(r_{1}\right) \leqq \int_{r_{1}}^{r_{2}} l^{*}\left(\left|r f^{\prime}\right|^{2}+1\right)^{1 / 2} d r=\int_{r_{1}}^{r_{2}} l^{*} \csc \psi^{*} d r .\left(^{8}\right)
$$

Here $l^{*}$ denotes the length of $S \cap \partial F^{*}$, that is $l^{*}=2 \pi r \sin f$, and, for each $x \in \partial F^{*}$ with $|x|=r>0, \psi^{*}=\psi^{*}(r)$ denotes the positive acute angle between the radius to $x$ and the normal to $\partial F^{*}$ at $x$, whenever the latter exists.

Next for each $x \in \partial F$ with $|x|>0$, let $\psi=\psi(x)$ be the corresponding angle between the radius to $x$ and the normal to $\partial F$ at $x$, whenever the latter exists. Then, because $\partial F$ is a polyhedral surface, it follows that

$$
\sigma\left(r_{2}\right)-\sigma\left(r_{1}\right)=\int_{r_{1}}^{r_{2}}\left(\int_{S \cap \partial F} \csc \psi d s\right) d r .
$$

Now fix $r, r+h \in J$ with $h>0$ and let $E$ be the central projection on $S$ of that part of $\partial F$ which lies in $r \leqq|x| \leqq r+h$. If $x \in S$ and if the radius through $x$ meets just one of the sets $S \cap F, S(r+h) \cap F$, then $x \in E$. Letting $\alpha$ denote the area of $E$ we thus obtain

$$
\alpha \geqq\left|A(r)-\left(\frac{r}{r+h}\right)^{2} A(r+h)\right|=\alpha^{*} .
$$

Since $\partial F$ is a polyhedral surface

$$
\lim _{h \rightarrow 0} \frac{\alpha}{h}=\int_{s \cap \partial F} \cot \psi d s
$$

for almost all $r$. Similarly from (14) it follows that

$$
\lim _{h \rightarrow 0} \frac{\alpha^{*}}{h}=l^{*}\left|r f^{\prime}\right|=l^{*} \cot \psi^{*}
$$

for almost all $r$, whence

$$
l^{*} \cot \psi^{*} \leqq \int_{S \cap \partial F} \cot \psi d s
$$

a.e. in $J$.

Finally for each $r \in J, S \cap \partial F$ bounds $S \cap F$, a set of area $A$. Hence applying the isoperimetric inequality on $S$, we conclude that the length of $S \cap \partial F$ is not less than the perimeter of the equivalent spherical cap $S \cap F^{*}$, that is

$$
l^{*} \leqq \int_{S \cap \partial F} d s{ }^{(4)}
$$

( ${ }^{3}$ We can prove equality here, but we do not use this fact.

(4) See $[9$, p. 90]. This is also a consequence of the theorem given on p. 233 of [1]. 
Applying Minkowski's inequality along with (17) and (18) yields

$$
\begin{aligned}
l^{*} \csc \psi^{*} & =\left(\left(l^{*} \cot \psi^{*}\right)^{2}+\left(l^{*}\right)^{2}\right)^{1 / 2} \\
& \leqq\left(\left(\int_{S \cap \partial F} \cot \psi d s\right)^{2}+\left(\int_{S \cap \partial F} d s\right)^{2}\right)^{1 / 2} \leqq \int_{S \cap \partial F} \csc \psi d s
\end{aligned}
$$

a.e. in $J$ and we obtain (13) from (15) and (16).

10. Spherical symmetrization of functions. The proof for Theorem 1 also requires that we introduce the notion of spherically symmetrized functions.

Let $u$ be everywhere continuous. We symmetrize $u$ to obtain a new function $u^{*}$ as follows. For each $a$, let $G_{a}$ and $F_{a}$ be the open and closed sets where $u<a$ and $u \leqq a$, respectively, and let $G_{a}^{*}$ and $F_{a}^{*}$ denote the spherical symmetrizations of these sets. Then given any point $x$, we see that $x \in F_{a}^{*}$ for sufficiently large $a$ and we define

$$
u^{*}(x)=\inf \left\{a \mid x \in F_{a}^{*}\right\} .
$$

It is then not difficult to verify that, for each $a, G_{a}^{*}$ and $F_{a}^{*}$ are precisely the sets where $u^{*}<a$ and $u^{*} \leqq a$, respectively. Since $G_{a}^{*}$ is open and $F_{a}^{*}$ closed, this means that $u^{*}$ is itself everywhere continuous. The following result shows that $u^{*}$ satisfies a Lipschitz condition whenever $u$ does. (Cf. Theorem 4.5 of [6].)

LEMma 4. If $u^{*}$ is the spherical symmetrization of $u$ and if

$$
\left|u\left(P_{1}\right)-u\left(P_{2}\right)\right| \leqq M\left|P_{1}-P_{2}\right|
$$

for all points $P_{1}$ and $P_{2}$, then

$$
\left|u^{*}\left(Q_{1}\right)-u^{*}\left(Q_{2}\right)\right| \leqq M\left|Q_{1}-Q_{2}\right|
$$

for all $Q_{1}$ and $Q_{2}$.

Proof. Fix two points $Q_{1}$ and $Q_{2}$ with $u^{*}\left(Q_{1}\right) \leqq u^{*}\left(Q_{2}\right)$ and let $a_{1}=u^{*}\left(Q_{1}\right)$ and $d=\left|Q_{1}-Q_{2}\right|$. For (19) it is sufficient to prove that

$$
u^{*}\left(Q_{2}\right) \leqq a_{2}=a_{1}+M d .
$$

Let $r_{1}=\left|Q_{1}\right|$ and $r_{2}=\left|Q_{2}\right|$, let $E_{1}$ be the set of points on $S_{1}=S\left(r_{1}\right)$ where $u \leqq a_{1}$, and let $E_{2}$ be the points on $S_{2}=S\left(r_{2}\right)$ whose distance from $E_{1}$ does not exceed $d$. Since $\left|r_{2}-r_{1}\right| \leqq d, E_{2}$ is clearly nonempty. $E_{2}$ is closed and for each $P_{2} \in E_{2}$ there exists a $P_{1} \in E_{1}$ such that $\left|P_{1}-P_{2}\right| \leqq d$. Thus

$$
u\left(P_{2}\right) \leqq u\left(P_{1}\right)+M d \leqq a_{2}
$$

and $u \leqq a_{2}$ at every point of $E_{2}$.

Next let $E_{1}^{*}$ and $E_{2}^{*}$ be the spherical symmetrizations of $E_{1}$ and $E_{2}$. Then $E_{1}^{*}$ is just the set of points on $S_{1}$ where $u^{*} \leqq a_{1}$ while $u^{*} \leqq a_{2}$ everywhere in $E_{2}^{*}$. Hence $Q_{1} \in E_{1}^{*}$ and to obtain (20) we need only show that $Q_{2} \in E_{2}^{*}$. We do 
this by appealing to the Brunn-Minkowski inequality for spherical geometry in the following manner.

Let $\alpha_{1} r_{1}$ and $\alpha_{2} r_{2}$ denote the radii of the closed spherical caps $E_{1}^{*}$ and $E_{2}^{*}$ measured along the spherical surfaces $S_{1}$ and $S_{2}$, respectively. If $H$ is the central projection of $E_{1}$ on $S_{2}$ and if we choose $\alpha$ so that

$$
0 \leqq \alpha \leqq \pi \text { and } d^{2}=r_{1}^{2}+r_{2}^{2}-2 r_{1} r_{2} \cos \alpha,
$$

then $E_{2}$ is just the union of the closed spherical caps with centers in $H$ and radii $\alpha r_{2}$ measured along $S_{2}$. Since $H$ has the same area as a spherical cap of radius $\alpha_{1} r_{2}$ on $S_{2}$, it follows from the aforementioned inequality that

$$
\alpha_{2} \geqq \min \left(\alpha_{1}+\alpha, \pi\right) .
$$

(See, for example, [9, p. 84].) Hence either $\alpha_{2}-\alpha_{1} \geqq \alpha$ or $\alpha_{2}=\pi$. In both cases it follows that $Q_{2} \in E_{2}^{*}$ and the proof is complete.

Lemma 4 can be used to derive an interesting geometrical property for spherically symmetrized rings. Let $R$ be a ring and define $u(x)$ as the distance from the point $x$ to $C_{0}$. Then $u=0$ on $C_{0}$,

$$
|u(P)-u(Q)| \leqq|P-Q|
$$

for all points $P$ and $Q$, and $u \geqq d$ on $C_{1}$ where $d$ is the distance between $C_{0}$ and $C_{1}$. Next let $R^{*}$ be the spherical symmetrization of $R$, let $C_{0}^{*}$ and $C_{1}^{*}$ be the components of $\mathrm{C}^{*}$, and let $u^{*}$ be the spherical symmetrization of $u$. Then $u^{*}=0$ on $C_{0}^{*}, u^{*} \geqq d$ on $C_{1}^{*}$ and Lemma 4 together with (21) yields

$$
d \leqq u^{*}(P)-u^{*}(Q) \leqq|P-Q|
$$

for all $P \in C_{1}^{*}$ and $Q \in C_{0}^{*}$. Thus $d \leqq d^{*}$, where $d^{*}$ is the distance between $C_{0}^{*}$ and $C_{1}^{*}$, and we conclude that the distance between the boundary components of a ring is not decreased under spherical symmetrization.

11. Equimeasurability. We also require the following property for spherically symmetrized functions in the proof for Theorem 1 .

Let $u^{*}$ be the spherical symmetrization of $u$, let $D$ and $D^{*}$ be the sets where $a_{1}<u<a_{2}$ and $a_{1}<u^{*}<a_{2}$, respectively, and let $f$ and $f^{*}$ be a pair of functions related to $u$ and $u^{*}$ as follows. For each $r \geqq 0$ and each $a_{1}<a<a_{2}$, $f$ and $f^{*}$ are constant and have the same value at the points of $S$ where $u=a$ and where $u^{*}=a$, respectively.

Lemma 5. If $f^{*}$ is continuous in $D^{*}$, then $f$ is continuous in $D$ and

$$
\int_{D}|f|^{a} d \omega=\int_{D^{*}}\left|f^{*}\right|^{a} d \omega
$$

for all $q>0$.

Proof. If $f$ is not continuous in $D$, we can find a $P \in D$ and a sequence 
$\left\{P_{n}\right\}$ in $D$ converging to $P$ such that

$$
\lim _{n \rightarrow \infty} f\left(P_{n}\right)=a \neq f(P) .
$$

Since $u$ and $u^{*}$ assume exactly the same values on $S \cap D$ and $S \cap D^{*}$, respectively, we can find a sequence $\left\{Q_{n}\right\}$ in $D^{*}$ such that $\left|Q_{n}\right|=\left|P_{n}\right|$ and $u^{*}\left(Q_{n}\right)$ $=u\left(P_{n}\right)$. Then a subsequence $\left\{Q_{n_{k}}\right\}$ will converge to a point $Q$ for which $|Q|=|P|$ and $u^{*}(Q)=u(P)$. Hence $Q \in D^{*}$ and

$$
f(P)=f^{*}(Q)=\cdot \lim _{k \rightarrow \infty} f^{*}\left(Q_{n_{k}}\right)=\lim _{k \rightarrow \infty} f\left(P_{n_{k}}\right)=a .
$$

This contradicts (23) and we conclude that $f$ is continuous in $D$.

We turn to the proof of (22). Fix $b$, let $G$ and $G^{*}$ be the sets where $f<b$ and $f^{*}<b$, respectively, and for each $r \geqq 0$ let $E$ be the set of values assumed by $u^{*}$ on $S \cap G^{*}$. Then, since $S \cap G^{*}$ is open on $S$ and $u^{*}$ is continuous, $E$ is the countable union of disjoint (possibly degenerate) linear intervals $I$. Next, since $u^{*}$ is the spherical symmetrization of $u$, the sets of points on $S$ for which $u \in I$ and $u^{*} \in I$ have equal area. Now $S \cap G$ and $S \cap G^{*}$ are just sets on $S$ which assign to $u$ and $u^{*}$, respectively, values in $E$ and, summing over the linear intervals $I$, we conclude that $S \cap G$ and $S \cap G^{*}$ have equal area.

Finally fix $b_{1}<b_{2}$ and let $G$ and $G^{*}$ be the sets where $b_{1} \leqq f<b_{2}$ and $b_{1} \leqq f^{*}$ $<b_{2}$. From the above we see that $S \cap G$ and $S \cap G^{*}$ have equal area and integration yields $m(G)=m\left(G^{*}\right)$. Thus $f$ and $f^{*}$ are equimeasurable functions and (22) follows directly. (See, for example, p. 277 of [5].)

12. Proof for Theorem 1 . We are given an arbitrary ring $R$ which we spherically symmetrize to obtain a second ring $R^{*}$. We want to prove that $\bmod R \leqq \bmod R^{*}$ or, alternatively, that

$$
\Gamma\left(R^{*}\right) \leqq \Gamma(R) .
$$

To establish (24) let $u$ be one of the simple admissible functions for $R$ discussed in $\$ 7$ and let $u^{*}$ be the spherical symmetrization of $u$. Since $\nabla u=0$ in all but a finite set of the tetrahedra $T, u$ satisfies a uniform Lipschitz condition. Hence, by Lemma 4 , the same is true of $u^{*}$. Now

$$
R^{*}=\left(R \cup C_{0}\right)^{*}-C_{0}^{*}
$$

where $\left(R \cup C_{0}\right)^{*}$ and $C_{0}^{*}$ are the spherical symmetrizations of the sets $R \cup C_{0}$ and $C_{0}$. Since $u$ is 0 on $C_{0}$ and 1 on $C_{1}, C_{0}$ is contained in the set where $u \leqq 0$ while $R \cup C_{0}$ contains the set where $u<1$. This together with the fact that $0 \leqq u^{*} \leqq 1$ implies that $u^{*}$ is 0 on $C_{0}^{*}$ and 1 on $C_{1}^{*}$. In particular we conclude that $u^{*}$ is admissible for the ring $R^{*}$.

The remainder of the argument is devoted to showing that

$$
\int_{R^{*}}\left|\nabla u^{*}\right|^{3} d \omega \leqq \int_{R}|\nabla u|^{3} d \omega
$$


For with (25) we obtain

$$
\Gamma\left(R^{*}\right) \leqq \int_{R}|\nabla u|^{3} d \omega
$$

and taking the infimum over all simple admissible functions $u$ yields (24) as desired.

Now let $0=a_{1}<a_{2}<\cdots<a_{n}=1$ be the finite set of values assumed by $u$ on the vertices of the tetrahedra $T$, and let $D_{i}$ and $D_{i}^{*}$ be the sets where $a_{i}<u<a_{i+1}$ and $a_{i}<u^{*}<a_{i+1}$, respectively. If the set where $u^{*}=a_{i}$ has positive measure, then almost all of its points are points of linear density in the directions of the coordinate axes. Since $\nabla u^{*}$ will vanish at almost all such density points, we conclude that the integral of $\left|\nabla u^{*}\right|^{3}$ over this set will vanish. Hence to establish (25) it suffices to show that

$$
\int_{D_{i}{ }^{*}}\left|\nabla u^{*}\right|^{3} d \omega \leqq \int_{D_{i}}|\nabla u|^{3} d \omega
$$

for $i=1, \cdots, n-1$.

Fix such an $i$, let $f^{*}$ be defined on $D_{i}^{*}$ and let $f^{*}$ be nonnegative, continuous and symmetric in the $x_{1}$-axis. That is, the value $f^{*}$ assumes at $x$ depends only on $|x|$ and $\phi$, the angle the radius to $x$ makes with the negative half of the $x_{1}$-axis. Next for each $a_{i}<a<a_{i+1}$, let $F, F^{*}$ denote the sets where $u \leqq a, u^{*} \leqq a$ and $\Sigma, \Sigma^{*}$ the sets where $u=a, u^{*}=a$. Then, as observed in $\S 7, F$ is a closed polyhedron and $\Sigma=\partial F$. It follows from this that $\Sigma^{*}=\partial F^{*}$ and that $f^{*}$ assumes exactly one value on each intersection $S \cap \Sigma^{*}$. We define a second function $f$ on $D_{i}$ by requiring that $f$ take this value on the corresponding intersection $S \cap \Sigma$.

Applying Lemma 5 we see that $f$ is continuous in $D_{i}$. We shall further show that

$$
\int_{D_{i}^{*}} f^{*}\left|\nabla u^{*}\right| d \omega \leqq \int_{D_{i}} f|\nabla u| d \omega .
$$

First let $\sigma=\sigma(a, r)$ and $\sigma^{*}=\sigma^{*}(a, r)$ denote the areas of the parts of $\Sigma$ and $\Sigma^{*}$ contained in $|x| \leqq r$. Then by Lemma 3,

$$
\sigma^{*}\left(a, r_{2}\right)-\sigma^{*}\left(a, r_{1}\right) \leqq \sigma\left(a, r_{2}\right)-\sigma\left(a, r_{1}\right)
$$

for $0 \leqq r_{1}<r_{2}$ and, since $f$ and $f^{*}$ are equal on corresponding intersections $S \cap \Sigma$ and $S \cap \Sigma^{*}$, we obtain

$$
\int_{\Sigma^{*}} f^{*} d \sigma^{*} \leqq \int_{\Sigma} f d \sigma .
$$

Now (28) holds for $a_{i}<a<a_{i+1}$ and, since $u$ and $u^{*}$ satisfy uniform Lipschitz conditions, we can apply a recent result due to Federer and Young to conclude that 


$$
\int_{D_{i}^{*}} f^{*}\left|\nabla u^{*}\right| d \omega=\int_{a_{i}}^{a_{i+1}}\left(\int_{\Sigma^{*}} f^{*} d \sigma^{*}\right) d a \leqq \int_{a_{i}}^{a_{i+1}}\left(\int_{\Sigma} f d \sigma\right) d a \int_{D_{i}} f|\nabla u| d \omega
$$

as desired. (See $[3$, p. 426].)

Now the function $\left|\nabla u^{*}\right|^{2}$ is bounded, measurable and symmetric in the $x_{1}$-axis. Hence we can find a sequence of functions $\left\{f_{n}^{*}\right\}$ which are nonnegative and continuous in $D_{i}^{*}$, symmetric in the $x_{1}$-axis and which converge boundedly to $\left|\nabla u^{*}\right|^{2}$ a.e. in $D_{i}^{*}$. Let $\left\{f_{n}\right\}$ be the corresponding sequence of functions defined on $D_{i}$ as above. Then (27) yields

$$
\int_{D_{i^{*}}}\left|\nabla u^{*}\right|{ }^{3} d \omega=\lim _{n \rightarrow \infty} \int_{D_{i^{*}}} f_{n}^{*}\left|\nabla u^{*}\right| d \omega \leqq \liminf _{n \rightarrow \infty} \int_{D_{i}} f_{n}|\nabla u| d \omega .
$$

Applying Hölder's inequality and (22) of Lemma 5 we obtain

$$
\begin{aligned}
\int_{D_{i}} f_{n}|\nabla u| d \omega & \leqq\left(\int_{D_{i}} f_{n}^{3 / 2} d \omega\right)^{2 / 3}\left(\int_{D_{i}}|\nabla u|^{3} d \omega\right)^{1 / 3} \\
& =\left(\int_{D_{i}^{*}}\left(f_{n}^{*}\right)^{3 / 2} d \omega\right)^{2 / 3}\left(\int_{D_{i}}|\nabla u|^{3} d \omega\right)^{1 / 3}
\end{aligned}
$$

and hence we conclude that

$$
\liminf _{n \rightarrow \infty} \int_{D_{i}} f_{n}|\nabla u| d \omega \leqq\left(\int_{D_{i}^{*}}\left|\nabla u^{*}\right|{ }^{3} d \omega\right)^{2 / 3}\left(\int_{D_{i}}|\nabla u|^{3} d \omega\right)^{1 / 3} .
$$

But (28) and (29) now imply (26) and the proof for Theorem 1 is complete.

\section{POINT SYMMETRIZATION}

13. Point symmetrization of rings. We consider next another kind of symmetrization which yields a second upper bound for the modulus of a ring.

Given an open set $G$ with $m(G)<\infty$ we define $G^{* *}$, the point symmetrizatlon of $G$, as the open sphere with center at the origin and volume equal to $m(G)$. For a closed set $F$ with $m(F)<\infty$, we take $F^{* *}$ as the closed sphere with volume $m(F)$; when $m(F)=0, F^{* *}$ will consist only of the origin.

Next let $R$ be a ring with $m(R)<\infty$. Then $R \cup C_{0}$ and $C_{0}$ are open and closed sets of finite measure and we define the point symmetrization of $R$ as

$$
R^{* *}=\left(R \cup C_{0}\right)^{* *}-C_{0}^{* *}
$$

The ring $R^{* *}$ is the spherical annulus which is metrically equivalent to $R$. We will establish the following space analogue of a theorem due to Carleman [2].

THEOREM 2. $\bmod R \leqq \bmod R^{* *}$.

The proof follows along the lines of the proof just given for Theorem 1 . However each step of the argument here is simpler than in the case of spheri- 
cal symmetrization. For example, the following analogue of Lemma 3 is now an immediate consequence of the classical isoperimetric property of the sphere.

LEMMA $3^{\prime}$. If $F$ is a closed polyhedron and if $F^{* *}$ is the point symmetrization of $F$, then the area of $\partial F^{* *}$ does not exceed that of $\partial F$.

We must introduce point symmetrized functions before considering the corresponding analogues for Lemmas 4 and 5 .

14. Point symmetrization of functions. Let $u$ be bounded above and continuous everywhere and let the set of points where $u<b=\sup u$ be of finite measure. Next for $a<b$ let $G_{a}$ and $F_{a}$ be the sets where $u<a$ and $u \leqq a$, respectively, and let $G_{a}^{* *}$ and $F_{a}^{* *}$ be the point symmetrizations of these sets.

We then define $u^{* *}$, the point symmetrization of $u$, as follows. Fix a point $x$. If $x \in F_{a}^{* *}$ for some $a<b$, we set

$$
u^{* *}(x)=\inf \left\{a \mid x \in F_{a}^{* *}\right\} .
$$

Otherwise we set $u^{* *}(x)=b$. It is easy to verify that $G_{a}^{* *}$ and $F_{a}^{* *}$ are the sets where $u^{* *}<a$ and $u^{* *} \leqq a$ for $a<b$, and then that $u^{* *}$ is everywhere continuous. We have also the following analogue for Lemma 4.

LEMMA $4^{\prime}$. If $u^{* *}$ is the point symmetrization of $u$ and if

$$
\left|u\left(P_{1}\right)-u\left(P_{2}\right)\right| \leqq M\left|P_{1}-P_{2}\right|
$$

for all $P_{1}$ and $P_{2}$, then

$$
\left|u^{* *}\left(Q_{1}\right)-u^{* *}\left(Q_{2}\right)\right| \leqq M\left|Q_{1}-Q_{2}\right|
$$

for all $Q_{1}$ and $Q_{2}$.

Proof. Fix two points $Q_{1}$ and $Q_{2}$ with $u^{* *}\left(Q_{1}\right) \leqq u^{* *}\left(Q_{2}\right)$ and let $a_{1}=u^{* *}\left(Q_{1}\right)$ and $d=\left|Q_{1}-Q_{2}\right|$. It is sufficient to show that

$$
u^{* * *}\left(Q_{2}\right) \leqq a_{2}=a_{1}+M d .
$$

If $a_{2} \geqq b$ there is nothing to prove. Hence we may assume that $a_{2}<b$.

Let $E_{1}$ be the closed set where $u \leqq a_{1}$ and let $E_{2}$ be the set of points whose distance from $E_{1}$ does not exceed $d$. From (30) it follows that $u \leqq a_{2}$ at every point of $E_{2}$. Now $E_{1}$ and $E_{2}$ correspond under point symmetrization to concentric closed spheres $E_{1}^{* *}$ and $E_{2}^{* *}$ of radii $r_{1}$ and $r_{2}$. Since $E_{1}^{* *}$ is the set where $u^{* *} \leqq a_{1}, Q_{1} \in E_{1}^{* *}$. The Brunn-Minkowski inequality for Euclidean geometry now implies that $r_{2} \geqq r_{1}+d$. (Again see $\left[9\right.$, p. 84].) Hence $Q_{2} \in E_{2}^{* *}$ and, since $u^{* *} \leqq a_{2}$ at all points of this set, we obtain (31) as desired( $\left.{ }^{6}\right)$.

(5) The Brunn-Minkowski inequality also shows directly that, as in the case of spherical symmetrization, the distance between the boundary components of a ring is not decreased under point symmetrization. 
15. Equimeasurability. For the analogue of Lemma 5, fix $a_{1}<a_{2} \leqq b$, let $D$ and $D^{* *}$ be the sets where $a_{1}<u<a_{2}$ and $a_{1}<u^{* *}<a_{2}$, respectively, and let $f$ and $f^{* *}$ be a pair of functions related to $u$ and $u^{* *}$ as follows. For each $a_{1}<a<a_{2}, f$ and $f^{* *}$ are constant and have the same value on the sets where $u=a$ and $u^{* *}=a$, respectively.

LEMMA $5^{\prime}$. If $f^{* *}$ is continuous in $D^{* *}$, then $f$ is continuous in $D$ and

$$
\int_{D}|f|^{a} d \omega=\int_{D^{* *}}\left|f^{* *}\right|^{a} d \omega
$$

for all $q>0$.

The proof for this result is similar to that for Lemma 5 and we omit it.

16. Proof for Theorem 2. We want to show that $\Gamma\left(R^{* *}\right) \leqq \Gamma(R)$. For this let $u$ be a simple admissible function for $R$. Since $m(R)<\infty$, the set of points where $u<1=\sup u$ is of finite measure and we let $u^{* *}$ be the point symmetrization of $u$. Then arguing as in $\S 12, u^{* *}$ is admissible for $R^{* *}$ and it remains only to show that

$$
\int_{R^{* *}}\left|\nabla u^{* *}\right|^{3} d \omega \leqq \int_{R}|\nabla u|^{3} d \omega
$$

Let $0=a_{1}<a_{2}<\cdots<a_{n}=1$ be the values assumed by $u$ on the vertices of the tetrahedra $T$, and let $D_{i}$ and $D_{i}^{* *}$ be the sets where $a_{i}<u<a_{i+1}$ and $a_{i}<u^{* *}<a_{i+1}$, respectively. As in $\$ 12$ it suffices to prove that

$$
\int_{D_{i} *^{* *}}\left|\nabla u^{* *}\right|^{3} d \omega \leqq \int_{D_{i}}|\nabla u|^{3} d \omega
$$

for $i=1, \cdots, n-1$.

Fix such an $i$, let $f^{* *}$ be defined in $D_{i}^{* *}$ and let $f^{* *}$ be nonnegative, continuous and symmetric in the origin. That is the value $f^{* *}$ assumes at $x$ depends only on $|x|$. Next for each $a_{i}<a<a_{i+1}$, let $F, F^{* *}$ be the sets where $u \leqq a, u^{* *} \leqq a$ and $\Sigma, \Sigma^{* *}$ the sets where $u=a, u^{* *}=a$. Then $\Sigma=\partial F, \Sigma^{* *}$ $=\partial F^{* *}$ and $f^{* *}$ assumes exactly one value on each level surface $\Sigma * *$. Define $f$ in $D_{i}$ by requiring that $f$ take this value on the corresponding level surface $\Sigma$. Then $f$ is continuous and, by virtue of Lemma $3^{\prime}$, we conclude that

$$
\begin{aligned}
\int_{D_{i} * *} f^{* *}\left|\nabla u^{* *}\right| d \omega & =\int_{a_{i}}^{a_{i+1}}\left(\int_{\Sigma^{* *}} f^{* *} d \sigma^{* *}\right) d a \leqq \int_{a_{i}}^{a_{i+1}}\left(\int_{\Sigma} f d \sigma\right) d a \\
& =\int_{D_{i}} f|\nabla u| d \omega .
\end{aligned}
$$

Finally arguing as in the last paragraph of $\$ 12$ we see that (34) and (32) of Lemma $5^{\prime}$ imply (33), thus completing the proof for Theorem 2. 
17. An upper bound for $\bmod R$. Theorem 2 now yields the following upper bound for the modulus of a ring.

THEOREM 3. Let $R$ be a ring. Then

$$
\bmod R \leqq \frac{1}{3} \log \frac{m\left(R \cup C_{0}\right)}{m\left(C_{0}\right)} .
$$

Proof. If $m(R)=\infty$, there is nothing to prove. Otherwise let $R^{* *}$ be the point symmetrization of $R$. Then $R^{* *}$ is the spherical annulus $a<|x|<b$, where $a$ and $b$ are chosen so that

$$
m\left(C_{0}\right)=\frac{4 \pi}{3} a^{3}, \quad m\left(R \cup C_{0}\right)=\frac{4 \pi}{3} b^{3} .
$$

Theorem 2 and (4) then imply that

$$
\bmod R \leqq \bmod R^{* *}=\log \frac{b}{a},
$$

from which (35) follows.

To obtain a similar bound by means of Theorem 1 we must first introduce a pair of extremal rings. They are the space analogues of rings studied by Grötzsch and Teichmüller.

\section{The GrötzsCh AND TeIChmÜller RINGS}

18. Definitions. For each $a>1$ we let $R_{G}=R_{G}(a)$ denote the ring whose complementary components consist of the sphere $|x| \leqq 1$ and the ray $a \leqq x_{1}$ $<\infty, x_{2}=x_{3}=0$. Similarly for each $b>0$ we let $R_{T}=R_{T}(b)$ denote the ring bounded by the segment $-1 \leqq x_{1} \leqq 0, x_{2}=x_{3}=0$ and the ray $b \leqq x_{1}<\infty$, $x_{2}=x_{3}=0$. Next, following Teichmüller [10], we set

$$
\bmod R_{G}=\log \Phi(a), \quad \bmod R_{T}=\log \Psi(b) .
$$

These functions have the following properties.

LEMMA 6. $\Phi(a) / a$ is nondecreasing in $1<a<\infty$ and

$$
\Psi(b)=\Phi\left((b+1)^{1 / 2}\right)^{2}
$$

for $b>0$.

Proof. For the first part fix $1<a<b$, let $R=R_{G}(b)$ and let $R^{\prime}$ and $R^{\prime \prime}$ be the two rings into which $R$ is split by $|x|=b / a$. Then (10) of Lemma 2 yields

$$
\log \Phi(b)=\bmod R \geqq \bmod R^{\prime}+\bmod R^{\prime \prime}=\log \frac{b}{a}+\log \Phi(a)
$$

whence $\Phi(b) / b \geqq \Phi(a) / a$ as desired. 
For the second part fix $b>0$, set $a=(b+1)^{1 / 2}$ and let $R$ be the ring bounded by the segment $0 \leqq x_{1} \leqq 1 / a, x_{2}=x_{3}=0$ and by the ray $a \leqq x_{1}<\infty, x_{2}=x_{3}=0$. Next let $R^{\prime}$ and $R^{\prime \prime}$ be the parts of $R$ contained in $|x|<1$ and $|x|>1$, respectively. Then $R^{\prime}$ and $R^{\prime \prime}$ are rings with equal moduli and Lemma 2 yields

$$
\log \Psi(b)=\bmod R \geqq 2 \bmod R^{\prime}=2 \log \Phi(a) .
$$

Hence to complete the proof for (36) it is sufficient to show that $\bmod R$ $\leqq 2 \bmod R^{\prime}$ or that

$$
\Gamma\left(R^{\prime}\right) \leqq 4 \Gamma(R) .
$$

Let $u$ be a continuously differentiable admissible function for $R$ and let $w=u+v$, where

$$
v=v(x)=1-u\left(\frac{x}{|x|^{2}}\right) .
$$

Then $w$ is admissible for $R^{\prime}$ and, since $|\nabla w(x)|=|x|^{-2}\left|\nabla w\left(x /|x|^{2}\right)\right|$,

$$
\int_{R^{\prime}}|\nabla w|^{3} d \omega=\int_{R^{\prime \prime}}|\nabla w|^{3} d \omega=\frac{1}{2} \int_{R}|\nabla w|^{3} d \omega .
$$

Minkowski's inequality now yields

$$
\begin{aligned}
\left(\int_{R}|\nabla w|^{3} d \omega\right)^{1 / 3} & \leqq \\
& \left(\int_{R}|\nabla u|^{3} d \omega\right)^{1 / 3}+\left(\int_{R}|\nabla \mathrm{v}|^{3} d \omega\right)^{1 / 3} \\
& =2\left(\int_{R}|\nabla u|^{3} d \omega\right)^{1 / 3}
\end{aligned}
$$

and we conclude from (38) that

$$
\Gamma\left(R^{\prime}\right) \leqq 4 \int_{R}|\nabla u|^{3} d \omega .
$$

Taking the infimum over all admissible $u$ gives (37) and the proof is complete.

19. Bounds for $\Phi(a)$. We derive here a pair of rough bounds for the function $\Phi(a)$. These, in turn, yield bounds for $\Psi(b)$.

The annulus $1<|x|<a$ separates the boundary components of $R_{G}$. Hence $\bmod R_{G} \geqq \log a$ and $\Phi(a) \geqq a$. Next $\Phi(a) / a$ is nondecreasing and approaches a limit $\lambda$ as $a \rightarrow \infty$. We will show that $\lambda$ is finite. This then gives the upper bound $\Phi(a) \leqq \lambda a$.

Let $R_{E}=R_{E}(a)$ denote the ring bounded by the segment $-1 \leqq x_{1} \leqq 1$, $x_{2}=x_{3}=0$ and by the ellipsoid

$$
\frac{x_{1}^{2}}{a^{2}+1}+\frac{x_{2}^{2}}{a^{2}}+\frac{x_{3}^{2}}{a^{2}}=1 .
$$


Next for $a>4$ let $R^{\prime}$ and $R^{\prime \prime}$ denote the rings bounded by the above segment and by the spherical surfaces with centers at $(-1,0,0)$ and radii $a-2$ and $a+2$, respectively. Then $R^{\prime}$ separates the boundary components of $R_{E}$ while $R_{E}$ separates those of $R^{\prime \prime}$. Hence

$$
\bmod R^{\prime} \leqq \bmod R_{E} \leqq \bmod R^{\prime \prime}
$$

and, since

$$
\bmod R^{\prime}=\log \Phi\left(\frac{a}{2}-1\right), \quad \bmod R^{\prime \prime}=\log \Phi\left(\frac{a}{2}+1\right)
$$

we conclude that

$$
\log \lambda=\lim _{a \rightarrow \infty}\left(\bmod R_{E}-\log \frac{a}{2}\right)
$$

Hence the problem is reduced to considering the asymptotic behaviour of $\bmod R_{E}$ as $a \rightarrow \infty$.

20. An inequality. In the case of two dimensions we know that, when $b=a+\left(a^{2}+1\right)^{1 / 2}$, the transformation

$$
y_{1}+i y_{2}=\frac{1}{2}\left(x_{1}+i x_{2}+\frac{1}{x_{1}+i x_{2}}\right)
$$

maps the plane ring $1<|x|<b$ conformally onto the ring bounded by the segment $-1 \leqq y_{1} \leqq 1, y_{2}=0$ and by the ellipse

$$
\frac{y_{1}^{2}}{a^{2}+1}+\frac{y_{2}^{2}}{a^{2}}=1
$$

We thus obtain the modulus for the plane analogue of the ring $R_{E}$.

The situation is more complicated in 3-space. Here we can show that a topological mapping (homeomorphism) preserves the moduli of rings if and only if it is conformal and that the only such mappings are the Moebius transformations. (See [4].) Hence for no number $b$ can we map $R$, the ring $1<|x|$ $<b$, conformally onto $R^{\prime}=R_{E}$, the ring bounded by $-1 \leqq y_{1} \leqq 1, y_{2}=y_{3}=0$, and by

$$
\frac{y_{1}^{2}}{a^{2}+1}+\frac{y_{2}^{2}}{a^{2}}+\frac{y_{3}^{2}}{a^{2}}=1 \text {. }
$$

On the other hand, when $a$ is large, there will exist numbers $b$ and mappings $y(x)$ of $R$ onto $R^{\prime}$ which are nearly conformal for large $|x|$. We prove a lemma which yields an upper bound for $\bmod R^{\prime}$ in terms of $\bmod R$ for such a mapping $y(x)$. 
LEMMA 7. Let $R$ be the ring $1<|x|<b$, let $y(x)$ be a topological mapping of $R$ onto a second ring $R^{\prime}$ and let $y(x)$ be continuously differentiable with nonvanishing Jacobian. Then

$$
\bmod R^{\prime} \leqq \bmod R+\int_{1}^{b}(D-1) \frac{d r}{r}
$$

where for $1<r<b$,

$$
D=D^{\prime}(r)=\max _{|x|=r}\left(\frac{I(x)^{3}}{J(x)}\right)^{1 / 2} .
$$

Here $J(x)$ denotes the absolute value of the Jacobian and $I(x)$ the maximum stretching at $x$, that is

$$
I(x)=\limsup _{x^{\prime} \rightarrow x} \frac{\left|y\left(x^{\prime}\right)-y(x)\right|}{\left|x^{\prime}-x\right|} .
$$

Proof. Let $v=v(y)$ be a continuously differentiable admissible function for $R^{\prime}$ and let $u(x)=v(y(x))$. Then integrating along a fixed radius yields

$$
1 \leqq \int_{1}^{b}|\nabla u| d r \leqq \int_{1}^{b}|\nabla v| I d r \leqq \int_{1}^{b}|\nabla v| D^{2 / 3} J^{1 / 3} d r
$$

and, with Hölder's inequality, we obtain

$$
1 \leqq\left(\int_{1}^{b}|\nabla v|^{3} J r^{2} d r\right)\left(\int_{1}^{b} D \frac{d r}{r}\right)^{2}
$$

Since this holds for all radii, we have

$$
4 \pi\left(\int_{1}^{b} D \frac{d r}{r}\right)^{-2} \leqq \int_{R}|\nabla v|^{3} J d \omega=\int_{R^{\prime}}|\nabla v|^{3} d \omega,
$$

and taking the infimum over all such functions $v$ gives

$$
4 \pi\left(\int_{1}^{b} D \frac{d r}{r}\right)^{-2} \leqq \Gamma\left(R^{\prime}\right)
$$

Hence

$$
\bmod R^{\prime} \leqq \int_{1}^{b} D \frac{d r}{r}=\bmod R+\int_{1}^{b}(D-1) \frac{d r}{r}
$$

and the proof is complete.

21. Estimate for $\lambda$. We now use this result to bound $\bmod R_{E}$ as follows. Introduce polar coordinates $(s, \alpha)$ and $(t, \beta)$ in the $x_{2} x_{3}$ and $y_{2} y_{3}$-planes, re- 
spectively. Next let $b=a+\left(a^{2}+1\right)^{1 / 2}$, let $R$ be the ring $1<|x|<b$, and define the mapping $y(x)$ as follows:

$$
y_{1}+i t=\frac{1}{2}\left(x_{1}+i s+\frac{1}{x_{1}+i s}\right), \quad \alpha=\beta .
$$

Then $y(x)$ maps $R$ onto $R^{\prime}=R_{E}$ and it is not difficult to verify that

$$
D^{2}=\max _{|x|=r} \frac{I(x)^{3}}{J(x)}=\frac{r^{2}+1}{r^{2}-1} .
$$

Hence, by Lemma 7,

$$
\bmod R_{E}<\bmod R+\int_{1}^{\infty}\left(\left(\frac{r^{2}+1}{r^{2}-1}\right)^{1 / 2}-1\right) \frac{d r}{r}=\log \lambda^{\prime} b,
$$

and elementary integration yields $\lambda^{\prime}=2^{1 / 2} e^{\pi / 4}$.

Finally (39) and (40) yield

$$
\log \lambda=\lim _{a \rightarrow \infty}\left(\bmod R_{E}-\log \frac{a}{2}\right) \leqq \lim _{a \rightarrow \infty} \frac{2 \lambda^{\prime} b}{a}=\log 4 \lambda^{\prime}
$$

and we obtain

$$
\lambda \leqq 4 \lambda^{\prime}=12.4 \cdots \cdot
$$

We have thus established the following rough bounds for $\Phi$.

LEMMA 8. $\Phi(a)$ satisfies the inequality

$$
a \leqq \Phi(a) \leqq \lambda a
$$

in $1<a<\infty$, where $\lambda$ is a finite constant, $\lambda \leqq 12.4 \cdots$.

22. Another upper bound for mod $R$. Finally combining Theorem 1 and Lemmas 6 and 8, we obtain a second upper bound for the modulus of a ring. It is the spherical symmetrization analogue of Theorem 3 and the space form of a theorem due to Teichmüller [10].

Theorem 4. Let $R$ be a ring and let $P$ be a point of $C_{0}$. If $C_{0}$ and $C_{1}$ contain points which lie at distances $a$ and $b$ from $P$, then

$$
\bmod R \leqq \log \Psi\left(\frac{b}{a}\right) \leqq \log \lambda^{2}\left(\frac{b}{a}+1\right)
$$

where $\lambda$ is the constant of Lemma 8 .

Proof. By performing a translation we may assume that $P$ is the origin. Next let $R^{*}$ be the spherical symmetrization of $R$. $C_{0}^{*}$ will contain the segment $-a \leqq x_{1} \leqq 0, x_{2}=x_{3}=0$ while $C_{1}^{*}$ will contain the ray $b \leqq x_{1}<\infty, x_{2}=x_{3}=0$. 
Hence $R^{*}$ separates the boundary components of the ring bounded by the above segment and ray. We conclude from Theorem 1 and Lemma 2 that

$$
\bmod R \leqq \bmod R^{*} \leqq \log \Psi\left(\frac{b}{a}\right),
$$

and the second inequality in (42) follows from (36) and (41).

\section{REFERENCES}

1. G. Bol, Isoperimetrische Ungleichungen für Bereiche auf Flächen, Jber. Deutsch. Math. Verein. vol. 51 (1941) pp. 219-257.

2. T. Carleman, Über ein Minimalproblem der mathematischen Physik, Math. Z. vol. 1 (1918) pp. 208-212.

3. H. Federer, Curvature measures, Trans. Amer. Math. Soc. vol. 93 (1959) pp. 418-491.

4. F. W. Gehring, Rings and quasiconformal mappings in space, Proc. Nat. Acad. Sci. U.S.A. vol. 47 (1961) pp. 98-105.

5. G. H. Hardy, J. E. Littlewood and G. Pólya, Inequalities, Cambridge University Press, 1934.

6. W. K. Hayman, Multivalent functions, Cambridge University Press, 1958.

7. C. Loewner, On the conformal capacity in space, J. Math. Mech. vol. 8 (1959) pp. 411-414.

8. G. Polya and G. Szegö, Isoperimetric inequalities in mathematical physics, Ann. of Math. Studies No. 27, Princeton University Press, 1951.

9. E. Schmidt, Die Brunn-Minkowskische Ungleichung und ihr Spiegelbild sowie die isoperimetrische Eigenschaft der Kugel in der euklidischen und nichteuklidischen Geometrie. I, Math. Nachr. vol. 1 (1948) pp. 81-157.

10. O. Teichmüller, Untersuchungen ïber konforme und quasikonforme Abbildung, Deutsche Math. vol. 3 (1938) pp. 621-678.

UNIVERSITY OF MichIGAN, ANn Arbor, Michigan 